\title{
The long unfinished march towards understanding microRNA-mediated repression
}

\author{
WITOLD FILIPOWICZ ${ }^{1}$ and NAHUM SONENBERG ${ }^{2}$ \\ ${ }^{1}$ Friedrich Miescher Institute for Biomedical Research, 4058 Basel and University of Basel, 4056 Basel, Switzerland \\ ${ }^{2}$ Department of Biochemistry and Goodman Cancer Research Center, McGill University, Montreal, Quebec, H3A 1A3 Canada
}

After 20 years, we can now really speak of a mature RNA. Congratulations!

The teens can be a time of hesitant development for a journal but leaving this period behind may also mean questions and difficult decisions: Should the format and focus remain the same or adjust to research trends? How can the competitive advantage to attract important papers in the field be maintained? Will the research area grow and remain exciting and relevant?

In particular the latter question should not worry a journal like RNA. In the mid-'90s, following the discoveries of mosaic genes and splicing, catalytic RNA, and RNA editing, it seemed rather unlikely that nature (and RNA) would continue to surprise us with even more secrets of comparable caliber. We were entirely wrong! The last 20 years have witnessed at least as many surprising discoveries in the RNA field as the two preceding decades. The parade of discoveries continued: RNA interference and diverse classes of small regulatory RNAs, pervasive transcription of metazoan genomes into thousands of long non-coding RNAs (lncRNAs), and RNAbased bacterial immunity CRISPR-Cas systems. In parallel, the development of many new sophisticated technologies, such as new-generation sequencing, single cell transcriptomics, as well as CLIP and ribosome foot-printing methodologies, has made it possible to study RNA-related phenomena at unprecedented depth and resolution.

One of the most remarkable discoveries of the last 20 years was the identification of thousands of microRNAs (miRNAs), 20-nt-long small RNAs acting as posttranscriptional regulators of gene expression. Since the discovery in 1993 of the first miRNA, lin-4 of Caenorhabditis elegans, miRNAs have remained contemporaries of RNA and, not surprisingly, $R N A$ has served as a forum for the publication of many important findings establishing the principles of miRNA function and metabolism.

Corresponding authors: witold.filipowicz@fmi.ch; nahum.sonenberg@ mcgill.ca

Article and publication date are at http://www.rnajournal.org/cgi/doi/ 10.1261/rna.051219.115. Freely available online through the RNA Open Access option.
Although understanding of the role played by miRNAs in eukaryotic physiology and human pathology has come a long way, mechanistic aspects of miRNA repression, in particular its translational component, remain unclear. In the last decade, we have both been heavily involved in studying the mechanism of miRNA-mediated repression of protein synthesis, generally in collaboration but occasionally as wellmeaning competitors racing to unravel a particular problem. Here, we review our research on miRNA-mediated repression and discuss recent developments and issues that need to be resolved. This commentary somewhat reflects our personal bias but the celebratory character of this issue of $R N A$ will surely allow a pinch of subjectivity. We apologize to colleagues whose work we do not mention, due to space limitations.

Research in Basel aimed at unraveling the mechanism of miRNA repression started in 2003. Our prior work on small RNA-mediated silencing had focused on the human Dicer protein and RNA interference (RNAi) in mammalian cells, including ES cells. In 2003, miRNAs were already known to be components of ribonucleoprotein (RNP) complexes, referred to as miRNPs or miRISCs, with Argonautes (AGOs) being the key protein fraction. Also, early studies from the laboratories of Ambros and Moss had indicated that mRNA targets of lin-4 in C. elegans remained associated with polysomes despite a marked reduction in the amounts of encoded proteins. Since the repressed mRNA levels did not seem to be significantly affected, these data pointed to translational elongation as a likely target of miRNAs. Ramesh Pillai, who arrived as a postdoc in 2003, decided to investigate whether miRNAindependent tethering of AGO proteins to mRNA mimics the repression in HeLa cells. Indeed, he found that tethering any of the three investigated human AGOs as phage $\lambda \mathrm{N}$-peptide fusions to the reporter 3 '-UTR bearing the $\mathrm{N}$-peptide-recognized boxB hairpins resulted in repression of protein synthesis without a major effect on mRNA levels. The data from this Really Nice Approach, published in 2004 in RNA (!),

(C) 2015 Filipowicz and Sonenberg This article, published in RNA, is available under a Creative Commons License (Attribution-NonCommercial 4.0 International), as described at http://creativecommons.org/licenses/ by-nc/4.0/ 
demonstrated formally that miRNAs just act as guides bringing repressive proteins to the mRNA. They also indicated that the specific architecture of the mRNA-miRNA interaction is required for the binding of miRNP to the target but not for the repression process per se, and that repression occurs primarily at the level of translation. It would be unfair to hide that we aimed originally to publish the results in a journal considered by some as even more prestigious than RNA (sorry RNA), but the referee's comment that "... publication will require demonstration of the repression mechanism by tethered AGOs ...", abruptly halted negotiations (Really Not Acceptable!).

To investigate in more detail and in a more physiological context how miRNAs inhibit protein accumulation in mammalian cells, Ramesh used mRNA reporters whose expression was regulated by endogenous let-7 miRNA. Repression of the reporters bearing three bulged let- 7 sites in their 3'UTR was accompanied by a strong shift of reporter mRNAs towards the top of a glycerol "polysome" gradient, similar to that seen upon addition of established inhibitors of translation initiation. Together with observations that repression was not associated with significant mRNA degradation and that translation of the $\mathrm{m}^{7} \mathrm{G}$-cap- but not IRESinitiated mRNAs was repressed, these results and others argued that miRNAs repress translation at the early step of initiation, involving cap recognition or another activity of the initiation factor eIF4F (trimeric complex, consisting of eIF4E, eIF4G, and eIF4A). Similar conclusions were reached independently by Preiss's lab.

The 2005 work of Ramesh addressed two further issues. Some early ideas and also later experimental data from Richter's lab raised the possibility that miRNPs affect protein accumulation by targeting nascent polypeptides for proteolysis. We found that reporters encoding proteins co-translationally targeted to endoplasmic reticulum (ER) were repressed by miRNAs or tethered AGO2, as for reporters encoding cytosolic proteins. This finding made the proteolysis model unlikely, a conclusion supported by high-throughput profiling data from Rajewsky's group, who found overrepresentation of mRNAs encoding ER proteins among mRNAs translationally repressed by miRNAs.

We also analyzed the intracellular localization of miRNP components and found that repressed mRNAs, AGO proteins, and miRNAs accumulate in processing bodies (PBs). These intracellular granules are enriched in translational repressors and mRNA catabolic enzymes, and are involved in storage and degradation of repressed mRNAs. Our data implicated these aggregates in miRNA-mediated silencing, as did similar independent observations from the laboratories of Hannon, Parker, and Chan. The latter further demonstrated that GW182 proteins (known as TNRC6 proteins in mammals) also localize to PBs. However, later work by Izaurralde and Rana showed that PBs, or at least their microscopically visible forms, are not essential for the repression, and that accumulation of repressed mRNAs in PBs is a consequence rather than a cause of repression. The fact that miRNA silencing can be recapitulated in cell-free extracts also suggests that repression is initiated outside of PBs.

Although the experiments discussed above suggested that repression of translation at early initiation steps is the main effect of miRNAs, evidence came to the fore in 2005-2006 that miRNAs also induce mRNA deadenylation and decay (the decay as a consequence of deadenylation). For many targets, either reporters or endogenous mRNAs, mRNA degradation explained most if not all of the effect on protein accumulation (work in the laboratories of Pasquinelli, Belasco, Izaurralde, Bartel, and Giraldez). This was further supported by later global studies combining SILAC proteomics or ribosome foot-printing with RNA-seq (Bartel, Rajewsky). In parallel, GW182/TNRC6 proteins recruited to miRNPs through direct interaction with AGOs became implicated in the mediation of the mRNA-destabilizing activity of miRNAs. Notably, at that time, steps in translation other than early initiation were also reported to be targets of miRNAs. For example, miRNA association with translating polysomes supported elongation as the repressed step (Nilsen, Richter). Further proposed mechanisms included the joining of the $60 S$ subunit during initiation and the premature termination or drop-off of elongating ribosomes. Consistently, some studies reported repression even when translation was driven by IRES elements. We refer Readers to Numerous Articles discussing the pros and cons of different early models.

In Basel, we were naturally concerned by the accumulating inconsistencies. Could this be due to repression by different paths, perhaps cell-, mRNA-, or miRNA-dependent? Although a definite answer to this question has not come by, we were reassured when Daniela Schmitter and Petr Svoboda in our lab and others (Preiss, Izaurralde) confirmed that the let-7 reporters previously used by us did not undergo pronounced miRNA-dependent degradation. Notably, in her 2006 publication, Daniela reported that another let-7 reporter used in parallel, but containing let-7 sites that base-pair to mRNA with a different geometry, was subjected to decay. Moreover, she found that miRNA reporters were more prone to decay when expressed in HEK293 than in HeLa and some other cells. In a further 2006 report from Basel, Suvendra Bhattacharyya showed that an endogenous CAT-1 mRNA was repressed by miR-122 in human hepatoma Huh7 cells without appreciable degradation. Suvendra also demonstrated that the repressed CAT-1 mRNA could be mobilized from $\mathrm{PBs}$ in response to stress and actively translated, i.e., that miRNA-mediated repression can be reversed under some conditions.

In the past, understanding of the translation mechanism and its regulation benefited greatly from biochemical approaches and the use of cell-free systems. A good example is the reconstitution of individual steps of translation in vitro using recombinant components and purified ribosomes or their subunits. In 2006, we initiated studies in Montreal aimed at the reconstitution of miRNA repression in vitro, 
working in close contact with Basel. By 2007, Geraldine Mathonnet, Marc Fabian, and Thomas Duchaine in Montreal reported the faithful recapitulation of mRNA repression in a cell-free translation system prepared from Krebs ascites cells. Remarkably, within the first 40 min of mRNA incubation in the extract, only $5^{\prime}$ cap-dependent translation was repressed by endogenous let-7 miRNAs, without mRNA degradation. Translation inhibition was relieved by adding the cap-binding protein complex, eIF4F, thus bolstering the former conclusion of Ramesh that the early step of cap-dependent initiation is the target of miRNAs. At the same time, other groups (Hentze, Wakiyama, Novina) used extracts from Drosophila embryos, HEK293 cells, and rabbit reticulocytes. The first two of these groups also concluded that miRNAs inhibit cap-dependent translation.

In a second Montreal study, Marc, Thomas and Geraldine demonstrated in 2009 that miRNA-mediated deadenylation can also be recapitulated in a cell-free extract of Krebs ascites cells. Using extracts biochemically depleted of different proteins that interact with miRNPs, they found that CAF1 (a component of the CCR4-NOT complex with established mRNA deadenylase activity) is essential for miRNA-mediated removal of poly(A) tails in vitro. Supplementation of the extract with wild-type CAF1, but not its catalytic mutant rescued the deadenylation. More unexpectedly, Marc and Geraldine also found that the poly(A)-binding protein $\mathrm{PABP}$ is required for miRNA-induced deadenylation. Dissection of the underlying mechanism revealed that PABP is recruited to the repressive complex by the C-terminaldomain of GW182/TNRC6 protein working downstream of AGO. The C-terminal TNRC6 region contains a sequence similar to the PAM2 motif present in several proteins known to interact with PABP. Work with recombinant protein fragments and their mutants demonstrated the involvement of the TNRC6 PAM2 in binding, and also the importance of the interaction for optimal deadenylation. The ensuing study by Marc in 2010, in collaboration with Doudna's group, established a crystal structure of TNRC6 PAM2 in a complex with the PABP fragment and amassed further evidence of the importance of the interaction for miRNA repression. Although the GW182 interaction with PABP is conserved in Drosophila and also in C. elegans, even though the GW182 equivalent in the worm lacks a recognizable PAM2 motif (Izaurralde), its precise role in miRNA repression and its generality are not entirely clear. Recent data indicate that PABP by binding to GW182 helps recruit miRNPs to mRNA, but the GW182-PABP interaction then facilitates PABP dissociation from the $\operatorname{poly}(\mathrm{A})$ tail (Hentze, Izaurralde). An alternative model proposes that binding of GW182 interferes with the interaction between PABP and the initiation factor $4 \mathrm{G}$ (eIF4G), which is known to be required for mRNA closed-loop formation and optimal translation.

Importantly, Marc and colleagues further demonstrated in 2009 that translational repression precedes mRNA deadenylation during the in vitro reaction. A similar conclusion emerged from later studies measuring the kinetics of miRNA action in cultured cells (Green, Filipowicz) or in vivo (Giraldez; but see Bartel's work in 2014 for an alternative interpretation of the zebrafish findings). For example, in 2011 in Basel, Julien Bethune found that translational repression is dominant up to $1-2 \mathrm{~h}$ post-induction in human cell lines stably expressing inducible reporters, followed by mRNA deadenylation and decay. Hence, it is perhaps not surprising that mRNA destabilization predominates the repression at the 12- to 48-h time-points of steady-state-like measurements in most of the global studies. Notably, in a recent 2014 global analysis of miRNA effects by ribosome footprinting and RNA-seq (Bartel's lab), in situations in which the early time-point measurements had reached statistical significance, repression of translation was dominant at $4 \mathrm{~h}$ post-miRNA-induction.

In recent years, attention has focused on the role of GW182/TNRC6 factors and proteins acting further downstream. Like other groups (e.g., Sonenberg, Chan, and particularly Izaurralde, whose laboratory has contributed greatly to this topic), in Basel we were dissecting both human (Jakob Zipprich) and Drosophila (Marina Chekulaeva, in collaboration with Parker) GW182/TNRC6s to establish the function of individual regions of these large 150 - to $200-\mathrm{kDa}$ proteins. They were known already to associate with Argonautes via the N-proximal Gly-Trp (GW) repeats (Ladurner, Izaurralde). As we reported in 2009 in two RNA papers, the C-terminal $\sim 400$ amino acid fragment (the C-terminal effector domain, CED) of human TNRC6s was sufficient to mediate repression similar to the whole protein. However, regions in the fly GW182 other than the CED also had strong repressive activity, particularly the $\mathrm{N}$-terminal portion of the protein (the $\mathrm{N}$-terminal effector domain, NED). In an extension of her work, Marina found that GW-like repeats present in the NED not only promote interaction with AGO proteins, but also mediate repression of protein synthesis. Although repression by the NED has been confirmed by others, the effect may not be general and may depend on the nature of the reporters used (Izaurralde).

How might GW-like repeats in GW182/TNRC6 proteins contribute to miRNA-mediated silencing? Marina Chekulaeva and Hansruedi Mathys in Basel investigated this problem using both human and Drosophila systems. They reported in 2011 that GW-like elements are not only present in Drosophila NED but also in repressive CEDs of the Drosophila and human GW182 proteins. Since Trp residues in these elements are flanked not only by Gly but also by Ser or Thr residues, the elements were named W-motifs to distinguish them from the AGO-recruiting GWs. We found that W-motifs, dispersed across the NED and CED domains and acting in an additive manner, recruit components of the CCR4-NOT and PAN2/PAN3 complexes that are known to be involved in mRNA deadenylation. The evidence that $\mathrm{W}$ motifs are not only necessary but also sufficient for repression was provided by gain-of-function experiments. A yeast 
protein fragment bearing engineered $\mathrm{W}$-motifs gained the ability to interact with CCR4-NOT and repress translation in the tethering assay. Interestingly, $\mathrm{W}$-motifs and recruitment of the CCR4-NOT complex also contributed to the repression of $\operatorname{poly}(\mathrm{A})^{-}$mRNAs; as this Repression was Not Associated with a decrease in mRNA level, it was most probably translational.

Experiments aimed at defining the contribution of the Cterminal portion of GW182 to miRNA repression were also carried out in Montreal. Marc Fabian demonstrated that this protein region (a silencing domain, SD) induces deadenylation in Krebs cell extracts when tethered to mRNA. Induction was the result of SD recruiting CCR4-NOT and PAN2/PAN2 deadenylases, as confirmed both in Krebs extracts and transfected HeLa cells. More detailed characterization of these SD regions identified two key elements, CIM-1 (C-NOT interacting motif) and CIM-2. Both contain conserved Trp residues, making it likely that their activity is Trp-dependent, as in the case of $\mathrm{W}$-motifs characterized in Basel. Interestingly, CIM-1 and CIM-2 elements were not entirely redundant: whilst CIM-2 supported complete deadenylation, CIM-1 only catalyzed partial removal of the poly(A).

The involvement of the SD/CED domain in the recruitment of CCR4-NOT and PAN2/PAN3 complexes was also demonstrated by Izaurralde's lab, and results from all three labs suggested that the SD/CED contacts the CNOT1 subunit of the CCR4-NOT. The CCR4-NOT is a complex of 10 evolutionarily conserved subunits, with the $\sim 250-\mathrm{kDa}$ CNOT1 protein acting as a scaffold binding the other subunits, including CAF1 deadenylase and CNOT9. Clearly, the next questions to address were: what is the molecular basis of W-motif-dependent interaction of GW182 CED/SD and CNOT1, and how does the CCR4-NOT1 complex, particularly CNOT1, contribute to miRNA-mediated repression of translation?

Hansruedi Mathys, working in Basel and closely collaborating with Elena Conti's group, identified three non-overlapping CNOT1 regions able to associate with the CED, with two of them (central CN9BD and the C-terminal NOT-SH) interacting in a $\mathrm{W}$-motif-dependent manner. Detailed characterization of the central interaction showed CED binding to CNOT1 to be mediated by CNOT9, which binds directly to the CED. X-ray analysis of the CNOT1 CN9BD complex with CNOT9 revealed that CNOT9 contains two potential Trp-binding pockets, the mutation of which eliminates interaction with the TNRC6 CED. Different regions of CNOT1 were also assayed for ability to repress tethered poly $(\mathrm{A})^{+}$and poly $(\mathrm{A})^{-}$mRNAs. A region encompassing the CN9BD and adjacent MIF4G (Middle domain of the initiation factor eIF4G) domain strongly inhibited both types of mRNA. In a search for mediators of the repressive effect, we identified the DEAD-box RNA helicase/ATPase DDX6 (also known as RCK/p54 in mammals and Dhh1 in yeast) as the MIF4G interactor, consistent with earlier findings of Rana implicating DDX6 in miRNA repression. Collaboration with Conti's lab was again very beneficial. Crystal structures of DDX6 in isolation and in complex with CNOT1 MIF4G revealed that interaction with MIF4G induces a dramatic change in the relative positioning of the two RecA domains of DDX6. Moreover, this conformational change was associated with strong activation of the ATPase activity of DDX6. Structure-based mutations and complementation assays showed that CNOT1-DDX6 interaction and DDX6 ATPase activity are important for miRNA-mediated repression. Moreover, further data offered support to the involvement of CNOT1-DDX6 interaction in the repression of translation (see below).

The function of DDX6 as a potential effector of miRNAmediated repression by CNOT1 was also studied by Chris Royua in Montreal. Through deletion analysis, he likewise identified CNOT1 MIF4G as the DDX6-interacting domain. Following in silico modeling of the interaction and mutant design, Chris reported in an RNA publication in 2014 that recruitment of DDX6 via CNOT1 is important for miRNA-mediated silencing. This was also established independently by the Izaurralde group, who also determined the structures of the CNOT1 CN9BD-CNOT9 complex containing the two Trp-binding pockets and the complex of CNOT1 MIF4G with the RecA2 domain fragment of DDX6.

The 2014 studies discussed above, converging on a role for DDX6 in miRNA silencing, revealed the multi-layered character of the repression process: base-pairing of AGO-miRNPs to the mRNA target, the consequent and consecutive recruitment of the GW182 proteins, the PAN2/PAN2 and CCR4NOT complexes, and finally DDX6. The high complexity of the process is further highlighted by the presence of multiple inhibitory regions in GW182s and that the CED, as the most important of these regions, can contact CNOT1 in three different ways, one being mediated by CNOT9. Moreover, dozens of proteins (e.g., FMRP, RACK1, EDD, Ataxin-2, HuR, and TRIM, LIM, and the Pumilio family proteins) have been identified as factors modulating the repression process, interacting either with miRNPs or targeted mRNAs. Knowing that expression of $>60 \%$ of all mammalian genes is predicted to be controlled by miRNAs, the complexity of the process is not that surprising. It reflects a potential to regulate miRNA silencing in many different ways, for example by modulating protein-protein interactions by post-translational modifications or by determining the intracellular location of the mRNA-miRNP assemblies.

But are we getting closer to comprehending all or most of the mechanistic details of miRNA-mediated silencing? The demonstration that GW182s directly recruit complexes such as CCR4-NOT and PAN2/PAN3 with deadenylase activity, and also associate with PABP, may lead us to conclude that the major players and interactions leading to mRNA deadenylation have been identified. But, in fact, precisely how the GW182-PABP interaction contributes to the process remains unclear. In marked contrast to deadenylation, many gaps remain in our grasp of the mechanism of translational 
repression. Several recent publications have implicated the eIF4A DEAD-box ATPases/helicases, components (together with eIF4E and eIF4G) of the eIF4F complex involved in $\mathrm{m}^{7} \mathrm{G}$ cap recognition and $5^{\prime}$-UTR scanning, as targets of the repression. Bushell's laboratory proposed that the CCR4-NOT complex recruits one of the two mammalian eIF4A paralogs, eIF4A2, to the repressed mRNA, possibly via the CNOT1 MIF4G domain. The eIF4A2 would then inhibit translation initiation by preventing the active eIF4A1 paralog from interacting with eIF4G, a process required for the $5^{\prime}$-UTR scanning. Two more recent papers from the Tomari and Fujiwara groups, provide data supporting an alternative conflicting model. These authors found in both mammalian and Drosophila systems that miRNPs interfere with the assembly of a functional eIF4F complex by displacing eIF4A from the mRNA. In the case of mammals, both eIF4A1 and eIF4A2 were displaced (in Drosophila, only one eIF4A form is expressed, which raises the question of how the "A2 contra A1" mechanism can operate in this organism). Ohlmanns' lab has also proposed the $5^{\prime}$-UTR scanning process as a target of miRNA repression.

Could in fact interference with eIF4A-mediated scanning and the inhibitory activity of DDX6 discussed earlier represent parts of the same repressive pathway? DDX6 has been shown to act as an inhibitor of translation initiation (Parker, Standart) or translation in general (Weis), and the in vitro study by Parker specifically identified $40 \mathrm{~S}$ recruitment as the affected initiation step, potentially consistent with a block in scanning. It is possible that DDX6 loaded onto mRNA via the interacting CNOT1 forms an obstacle to eIF4A progress along the $5^{\prime}$-UTR, leading to its dissociation from the mRNA. DDX6 might also remodel the initiation complex at the $5^{\prime}$-UTR; the protein was reported to interact with cap-binding protein eIF4E and also eIF4E-T, an eIF4E-binding protein, which blocks its association with eIF4G. But very different scenarios are also possible. DDX6 is a very abundant protein with the potential to oligomerize along RNA (Weil, Standart). This property could lead to inhibition of the elongation process, consistent with a report that DDX6 in yeast can slow ribosome movement (Coller). A further plausible scenario invokes a role for DDX6 in nucleating mRNA decapping. DDX6 is known to interact directly with decapping activators such as Pat1, Edc3, and RAP55/TraI (Izaurralde, Conti).

These considerations bring us back to the issue of just how many different mechanisms that mediate miRNA function actually operate in cells. It is reasonable to expect that deadenylation per se involves a single mechanism, with PAN2/ PAN3 initiating poly(A) removal and CCR4-NOT completing it (Shyu), whilst the initial activation of the process may possibly occur in many different ways. With regard to miRNA effects on translation, in the light of the enormous complexity of the translation process itself, particularly its initiation, it would be surprising if miRNAs exert their effects by just one conserved mechanism. In support of this, other mechanisms proposed Recently Now Add to the mechanisms that emerged from the early studies and those discussed above. For example, Tomari's group has reported that AGO1-miRNP in Drosophila embryo lysates can induce translational repression at an early step of initiation in a GW182dependent or GW182-independent way. Carthew's group has also identified AGO1-miRNP complexes in Drosophila cells that repress translation in a GW182-dependent or -independent manner. However, the GW182-deficient complex, regulated by mitogenic signaling, appeared to target translation elongation. Among the diverse miRNA effects are also those that lead to the stimulation rather than the inhibition of translation. Activation of mRNA translation by miRNAs, first reported by Vasudevan and Steitz, occurs, for example, in quiescent cells arrested in G0.

Of the dozen or so reported miRNA-induced translational effects, none is fully understood at the molecular level. Biochemical and structural studies are needed to define the interactions between individual components and the dynamics of formation of complexes involved in different pathways. The future will show which of the proposed mechanisms stands the test of time. We also have a very limited understanding of how the dozens of proteins implicated in regulating miRNP activity (positively or negatively) really work; some of these proteins are listed above. Regrettably, many of the initial publications claiming the identification of regulatory factors were not followed up by further, more detailed biochemical and structural studies that might explain the mechanism or the generality of their involvement.

One issue that continues to generate much debate is the temporal order of the repressive events. Which is first (and more important!): repression of translation or mRNA deadenylation? Although kinetic studies demonstrated that translational inhibition precedes mRNA deadenylation, there are no data confirming that repression of translation is a precondition for deadenylation to occur. Hence, it is possible that the observed sequence of events is due, for example, to more rapid assembly of complexes repressing translation than those initiating deadenylation. Notwithstanding, it is likely that both processes are frequently initiated simultaneously and independently; as soon as both are up and running, translational repression will bolster deadenylation and vice versa. The finding that the same complex, CCR4-NOT, mediates both translational repression and deadenylation offers support to this notion. What is clear, and supported by many cellular and most in vitro studies, is that repression of translation is an integral component of miRNA-mediated silencing. It is also clear that at steady state, mRNA degradation explains most of the effects on protein accumulation for a majority of miRNA targets. However, exceptions to this "almost a rule" situation exist, as discussed above and also as shown in some global studies (e.g., the SILAC 2008 study of Rajewsky; see also our 2008 review).

On a hopeful note, recent years have witnessed considerable progress in understanding some structural aspects of 
miRNA repression. Crystallographic studies have provided much information about the loading of miRNAs and other small RNAs onto AGO proteins and the interaction of the resulting complexes with target RNAs (Patel, MacRae). Moreover, the 2012 study of MacRae, identifying two Trpbinding pockets in human AGO2, provided the first insight into how GW repeats might mediate protein-protein interactions, in this case the binding of GW182 proteins to Argonuates. This was followed by identification of the Trp-binding pockets in PAN2/PAN3 and CNOT9 responsible for $\mathrm{W}$-motif-dependent recruitment of PAN2/PAN3 and CCR4-NOT complexes by GW182s, respectively (Izaurralde, Conti/ Filipowicz). It will be interesting to find out how individual GW repeats or $\mathrm{W}$-motifs, which are quite similar, discriminate between their different targets. Further structural studies discussed above have provided details of the interaction of GW182s with PABP and of the CNOT1 protein with
CNOT9 and DDX6, all these interactions being relevant to miRNA-mediated silencing. Since structural information allows the design of specific mutations to test the relevance of different interactions, it is hoped that future structural studies, possibly combined with robust in vitro systems, will greatly improve our understanding of miRNA silencing.

In the past decade, we have both been deeply involved in studies of miRNAs. This has been one of the most interesting episodes of our scientific carriers and has sustained our relationship over 30 years in science and beyond. Not only have we both greatly enjoyed hundreds of inspiring telephone conversations (the phone companies probably enjoyed them even more), but we have taken great pleasure in sharing our interest in RNA with dozens of colleagues and friends, and-for sure-in reading every month a new issue of $R N A$. Thank you, Tim for keeping the RNA/RNA fire burning so bright. 

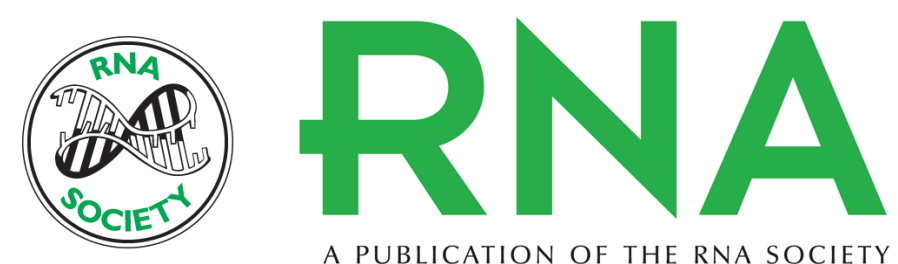

A PUBLICATION OF THE RNA SOCIETY

\section{The long unfinished march towards understanding microRNA-mediated repression}

Witold Filipowicz and Nahum Sonenberg

RNA 2015 21: 519-524

Open Access Freely available online through the RNA Open Access option.

Creative This article, published in $R N A$, is available under a Creative Commons License

Commons (Attribution-NonCommercial 4.0 International), as described at

License http://creativecommons.org/licenses/by-nc/4.0/.

Email Alerting Receive free email alerts when new articles cite this article - sign up in the box at the Service top right corner of the article or click here. 